\title{
Full recovery of Akinetic Mutism after corpus callosum infarction in post subarachnoid hemorrhage of ruptured distal anterior cerebral artery aneurysm
}

Ibrahim Alnaami, $M S c, F R C S C$, Saeed A. Alqabtani, $M D, A B P N$.

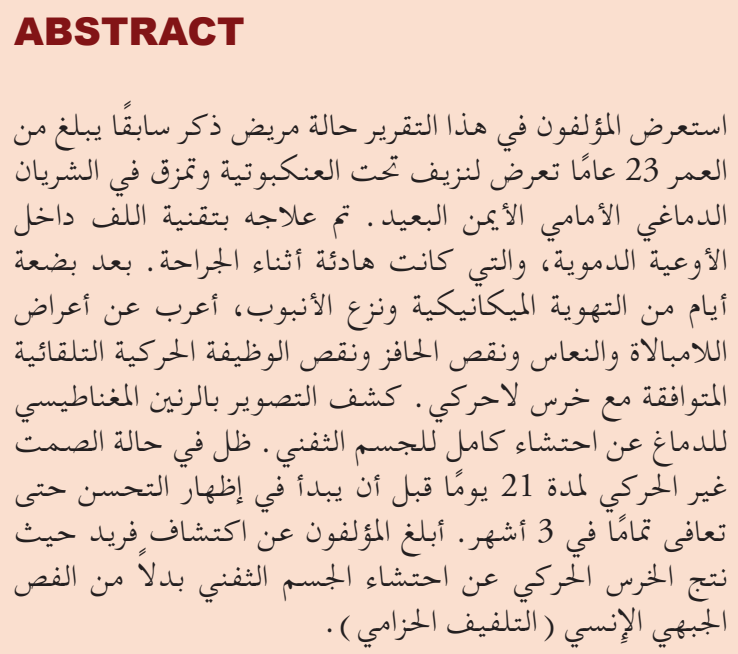

The authors report a previously healthy 23-yearold male patient who presented with subarachnoid hemorrhage and was found to have a ruptured right distal anterior cerebral artery aneurysm. He was treated by endovascular coiling technique, which was uneventful perioperatively. After a few days of mechanical ventilation and upon extubation, he expressed symptoms of apathy, drowsiness, lack of motivation, and lack of spontaneous motor function consistent with akinetic mutism. The magnetic resonance imaging of the brain revealed infarction of the whole body of corpus callosum. He remained in akinetic mutism status for twenty-one days before he started to show improvement until he fully recovered in 3 months. The authors report a unique finding where akinetic mutism resulted from infarction of the corpus callosum rather than medial frontal lobe (cingulate gyrus).

Neurosciences 2020; Vol. 25 (5): 412-415 doi: 10.17712/nsj.2020.5.20200071

Division of Neurosurgery (Alnaami), Department of Surgery, Division of Neurology (Alqahtani), Department of Medicine, King Khalid University, Abha, Kingdom of Saudi Arabia
Received 11th May 2020. Accepted 13th July 2020.

Address correspondence and reprint request to: Dr. Ibrahim Alnaami, Division of Neurosurgery, Department of Surgery, College of Medicine, King Khalid University, Abha, Kingdom of Saudi Arabia

E-mail:ialnaami@gmail.com

ORCID ID: https://orcid.org/0000-0001-6433-1990

A kinetic mutism (AM) is a syndrome of psychiatric, verbal, and motor components. It is a state of limited responsiveness to the environment in the absence of gross alteration of sensorimotor mechanisms operating at a more peripheral level. Neither paralysis nor coma accounts for the symptoms. ${ }^{1,2}$ The AM may result from trauma, infarctions, infections or others. AM have been rarely reported as a consequence of aneurysmal subarachnoid hemorrhage. The authors report a case of young male patient who is previously healthy, who presented with aneurysmal SAH. He developed AM as a result of corpus callosum infarction without involvement of cingulate gyrus. The novel finding in our study is that infarction did not affect the cingulate gyrus nor medial frontal lobes. In fact, the insult was limited to the body of corpus callosum only, sparing both the genu and the splenium.

Case Report. Patient information and clinical presentation. A previously healthy 23 -year-old male patient who presented to the Emergency Room with sudden onset of excruciating headache. On initial assessment, his Glasgow coma score (GCS) was 14 over 15 , with equal reactive pupils bilaterally and no apparent neurological deficit.

Disclosure. Authors have no conflict of interests, and the work was not supported or funded by any drug company. 


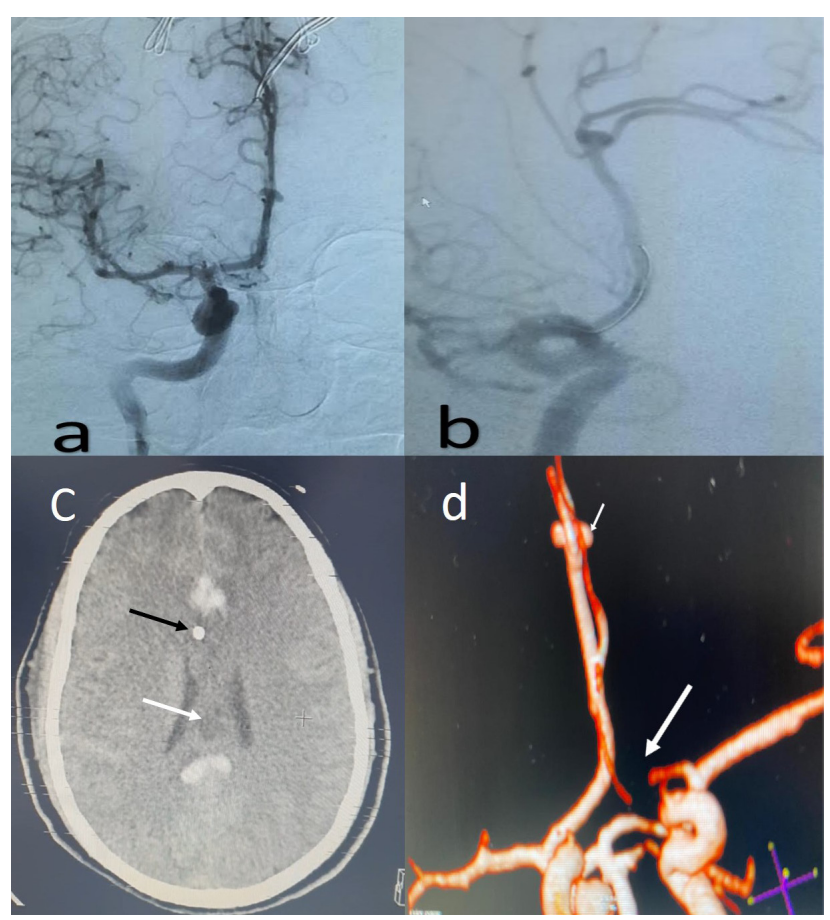

Figure 1 - Digital subtraction angiography a) AP view and b) oblique view of right internal carotid artery injections depicting right DACA aneurysm. c) axial computed tomography of the brain prior to coiling andafter external ventricular drain being inserted (black arrow) showed small area of hypodensity in corpus callosum (white arrow). d) 3D CT angiography of cerebral vessels in oblique view, reveled hypoplastic left anterior cerebral artery (long white arrow) and the aneurysm (short white arrow).

Diagnostic assessment. In the peripheral hospital, brain computed tomography (CT) revealed extensive subarachnoid hemorrhage (SAH) with no significant hydrocephalus. While completing his workup in the emergency department, his level of consciousness rapidly dropped to 8 over 15 with no apparent seizure attack. He was immediately intubated for airway protection and started on sedation. The brain CT was repeated immediately to reassess for presumed aneurysm re-rupture or obstructive hydrocephalus.

Therapeutic intervention. The repeated image did not reveal new bleed or worsening of hydrocephalus. However, the neurosurgeon elected to insert an external ventricular drain for monitoring and preparation of transfer to another health-care facility for definitive treatment. The cerebral computed tomography angiogram (CTA) revealed a $5 \times 4 \times 4 \mathrm{~mm}$ bilobed right distal anterior cerebral artery aneurysm (DACA) (pericallosal aneurysm).

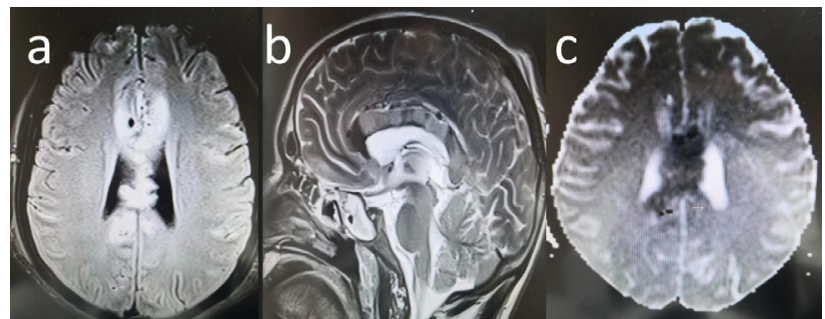

Figure 2 - Post coiling MRI a) axial T1 FLAIR b) sagittal T2, and c) axial ADC sequence MRI of the brain revealed infarction of the whole body of corpus callosum.
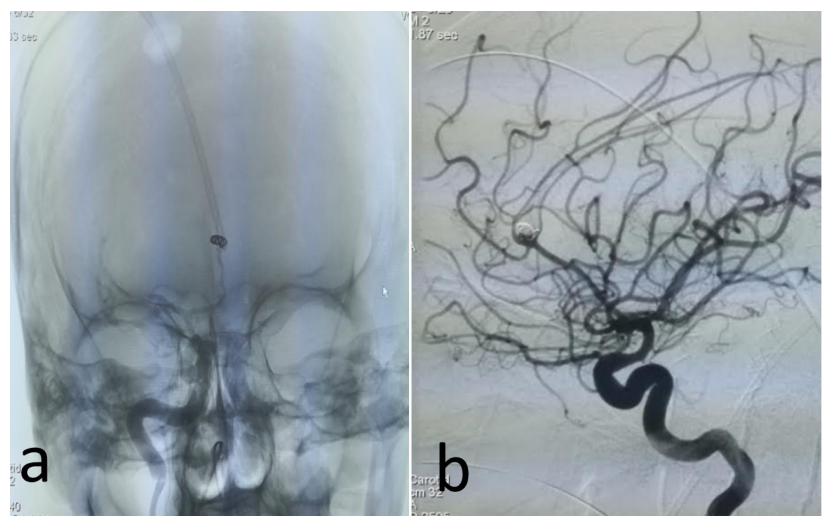

Figure 3 - follow-up angiogram a) AP view shows coils mass inside the aneurysm, b) and lateral internal carotid artery injection depicting embolized aneurysm with mild vasospasm in the distal anterior cerebral artery.

After arrival at our hospital, the patient was moving all his limbs and opening his eyes only to painful provocation. He was taken urgently to the angiography suite. The aneurysm was occluded successfully using the coiling technique, and there were no intraoperative complications (Figure 1).

Follow-up and outcome. Postoperatively, he received standard Intensive Care Unit supportive therapy for a few days. The neurosurgery team and ICU made the patient extubated. Upon extubation, the neurological examination showed that patient eyes were closed. He did not speak spontaneously. With the painful stimulation, the patient opened his eyes. He uttered a few slurred and dysarthric words. In the next few days, he started to obeying single step commands and opened eyes spontaneously. He was awake, but the patient expressed signs of apathy. He demonstrated a lack of motivation, indifference, and a scarcity of facial and emotional expressions when interacted with a close family member during the ICU visits. No abnormal movements observed, and no grasp reflexes noticed. Over the next 3 weeks, he had spontaneous speech but 


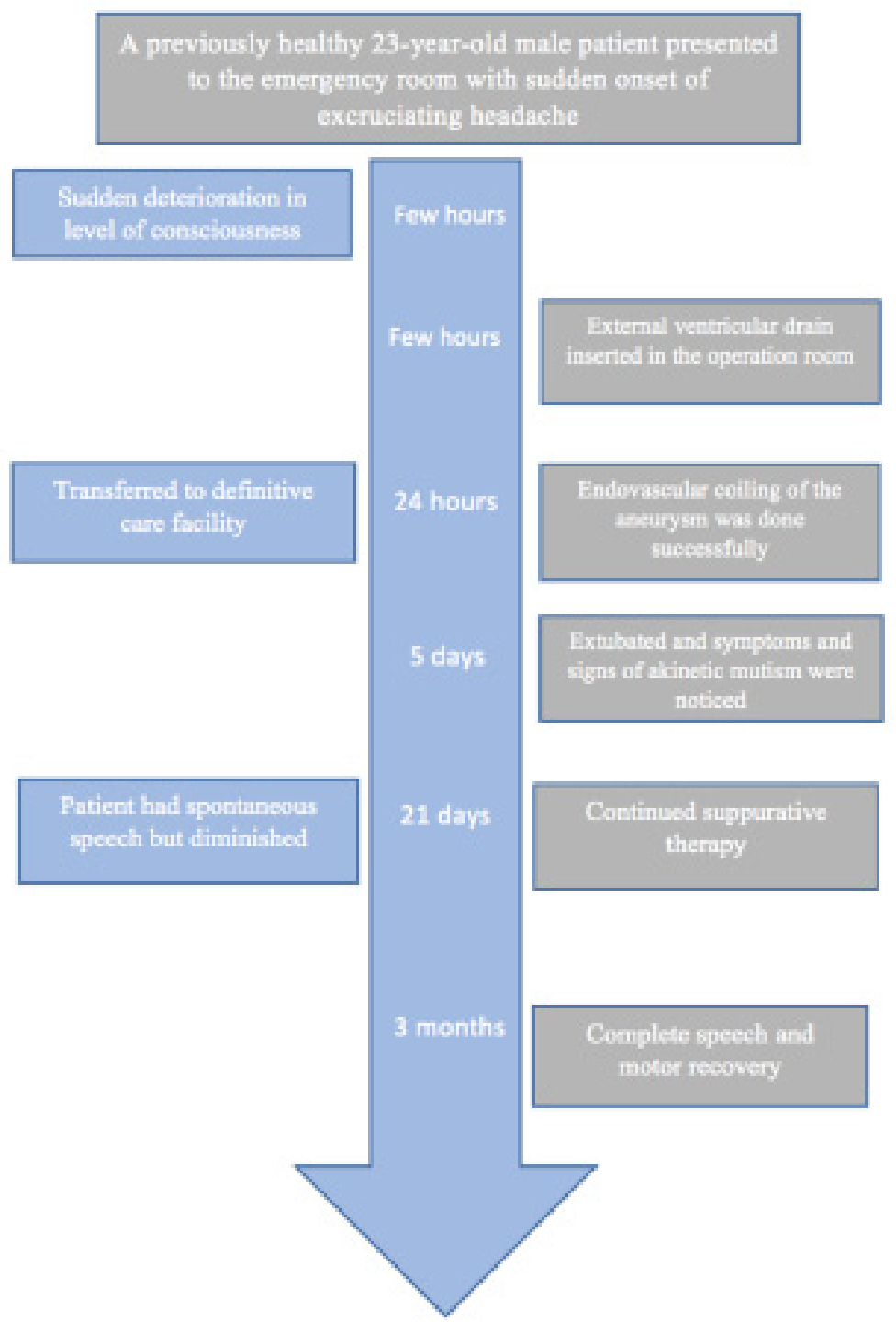

Figure 4 - Timeline of the course of the management of 23-year-old male who developed akinetic mutism after rupture of distal anterior cerebral artery aneurysm.

diminished. He started to have a normal spontaneous movement in all 4 extremities within the context of preserved alertness.

Magnetic resonance imaging (MRI) of the brain showed infarction of the entire body of corpus callosum (Figure 2). The patient underwent multiple visits to the angiography suite to check for any possibility of vasospasm; however, a repeated cerebral angiogram revealed mild vasospasm in distal anterior cerebral arteries branches (Figure 3).

Clinically, the patient was not in a coma or decreased consciousness; he remained in the akinetic mutism status for 21 days. We believe that he was in akinetic mutism due to the persistent presence of apathy, abulia, lack of motivation, and emotional response, along with the initial lack of spontaneous speech with preserved wakefulness. The patient then started to gradually recover, regaining full speech first then motor function, until he had complete recovery after three months.

Discussion. Aneurysmal subarachnoid hemorrhage is a well-known medical condition with devastating consequences such as vasospasm, seizures, 
hydrocephalus, and others. One of its rarely documented consequences is akinetic mutism.

Akinetic mutism is a syndrome of psychiatric, verbal, and motor components. It is a state of limited responsiveness to the environment in the absence of gross alteration of sensorimotor mechanisms operating at a more peripheral level. Neither paralysis nor coma accounts for the symptoms. ${ }^{1,2}$

The patients with akinetic mutism cannot speak or move. These deficits do not result from a direct effect on motor or speech fibers per se, but from the lack of neuronal initiative circuitry. From the neuroanatomical point of view, this syndrome has been classified into telencephalic, where medial frontal lobes are affected, diencephalic in which thalamus and basal ganglia are affected, and mesencephalic, where the upper brainstem is also affected.

Akinetic mutism may result from trauma, infections, infarction, carbon monoxide poisoning, hydrocephalus, post transcallosal approach, ${ }^{2}$ and others. However, the first time akinetic mutism was linked to $\mathrm{SAH}$ was in $1971 .^{2,3}$

In 2004, Choudhary concluded the dismal prognosis of akinetic mutism in post SAH patients. Of the 21 patients reviewed in that study, 2 only had a distal ACA aneurysm. Later, a few other papers reported akinetic mutism in DACA. ${ }^{3-5}$ Apart from the report by Sibille et $\mathrm{al}^{6} 2016$ all cases of akinetic mutism post SAH in DACA had a poor prognosis, where patients ended up with significant disabilities and being dependent or in vegetative states.

The characteristic finding in the reports above is the infarction of the medial frontal lobe (cingulate gyrus) or the presence of hydrocephalus. The novel finding in our study is that infarction did not affect the cingulate gyrus nor medial frontal lobes. In fact, the insult was limited to the body of corpus callosum only, sparing both the genu and the splenium.

The mechanism in which the authors speculate the full recovery of the patient's akinetic mutism is attributed to the fact that only the body of the corpus callosum was affected. The patient course was similar to the transient mutism that results from callosotomy procedures in refractory epilepsy patients where their mutism may last up to twenty-five days. ${ }^{6}$ The patient's age is also considered a favorable prognostic factor.

In conclusion, the authors report a unique finding where akinetic mutism resulted from infarction of the corpus callosum rather than medial frontal lobe (cingulate gyrus), which followed a rupture of DACA aneurysm, and a rare complete recovery for such pathology, where young patient's age played an important role in this outcome.

Acknowledgement. The authors would like to acknowledge ProofReadingService.com (www.proofreadingservice.com) for English language editing.

\section{References}

1. Burst JCM, Chamorro A. Anterior cerebral artery disease. In: Grotta JC, Albers GW, Broderick JP, Kasner SE, Lo EH, Mendelow AD, editors. Stroke: Pathophysiology, diagnosis, and management. Elsevier; 2016. p 347-361.

2. Nakasu Y, Isozumi T, Nioka H, Handa J. Mechanism of mutism following the transcallosal approach to the ventricles. Acta Neurochir 1990; 110: 146-153.

3. Choudhari KA. Subarachnoid haemorrhage and akinetic mutism. Br J Neurosurg 2004; 18: 253-258.

4. Herklots MW, Oldenbeuving A, Beute GN, Roks G, Schoonman GG. Lack of motivation: Akinetic mutism after subarachnoid haemorrhage. Netherlands Journal of Critical Care 2015; 24: 29-32.

5. Yoon SM, Chun, YI, Kim WK. Akinetic mutism following spontaneous subarachnoid hemorrhage-A case report. Korean Journal of Cerebrovascular Surgery 2008; 10: 335-339.

6. Sibille FX, Hantson P, Duprez T, van Pesch V, Giglioli S. Reversible akinetic mutism after aneurysmal subarachnoid haemorrhage in the territory of the anterior cerebral artery without permanent ischaemic damage to anterior cingulate gyri. Case Rep Neurol Med 2016; 2016: 5193825.

7. Quattrini A, Pesce MD, Provinciali L, Cesarano R, Ortenzi A, Paggi A, et al. Mutism in 36 patients who underwent callosotomy for drug-resistant epilepsy. J Neurosurg Sci 1997; 41:93-96. 